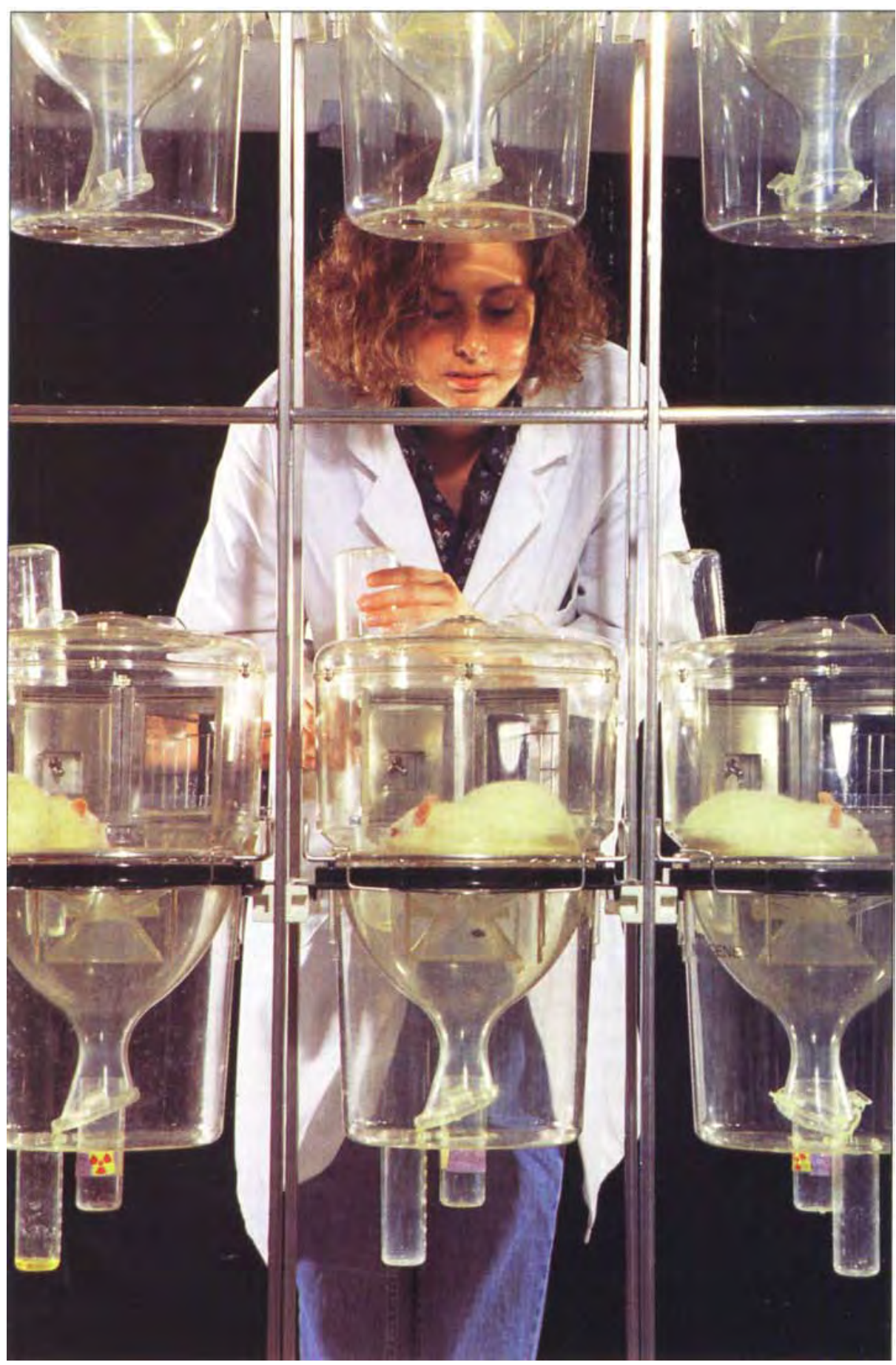

Results from animal metabolism studies provide information to assess the toxicological effects of chemicals. UC Davis scientists are determining how rapidly rats eliminate pesticides from their bodies and whether toxic metabolites are formed.

Dietary pesticide risk assessment is an imprecise process requiring a series of judgments based on both scientific and philosophical grounds. At best, it is a crude quantitative tool to prioritize risks and allocate resources - a method involving a great deal of uncertainty. Where uncertainties exist, scientists make conservative assumptions designed to increase the risk estimate so that errors are made on the side of safety.

While risk assessment plays a necessary and critical role in pesticide regulation, the uncertainty inherent in the process must be appreciated if appropriate science-based policies are to be developed. Scientific advances are needed to improve risk assessment accuracy, and policies must be flexible enough to allow such advances to be incorporated into the risk assessment process.

\title{
Lawmakers should recognize uncertainties in risk assessment
}


$\mathrm{T}$ he National Academy of Sciences (NAS) report Pesticides in the Diets of Infants and Children focused national attention on the process of assessing dietary risk from pesticides. A major conclusion of the report was that significant improvements in risk assessment are necessary if regulators are to ensure a safe food supply for all consumers, including sensitive subgroups such as infants and children. However, the report did not suggest that infants, children or adults are now at excessive risk from pesticides in the diet.

While risk assessments are developed to protect public health, they also have economic repercussions for consumers and the agricultural community. They are the basis for regulatory and legislative policies determining under which circumstances a particular pesticide's use is allowed. These, in turn, may influence the availability and cost of food items and may have consequences in foreign food production practices and the regulation of international trade, since exporters of food into the United States are subject to the same standards as domestic producers.

At the present time, risk assessment methods are complicated and controversial, and results of risk assessments may be subject to a variety of interpretations. This paper examines risk assessment and the potential impact of the NAS committee recommendations, suggests guidelines for the interpretation of risk assessments and discusses the scientific challenges and policy implications of the risk assessment process.

\section{Risk assessment: an overview}

The risk assessment process consists of hazard identification, dose-response assessment, exposure assessment and risk characterization (see fig. 1 and box, p. 23). The first two steps constitute the toxicological assessment phase, which generally consists of extrapolation of the results of animal toxicology studies to determine the potential for human effects at specific levels of exposure and evaluation of the quality of the toxicology data. The exposure assessment step requires es-

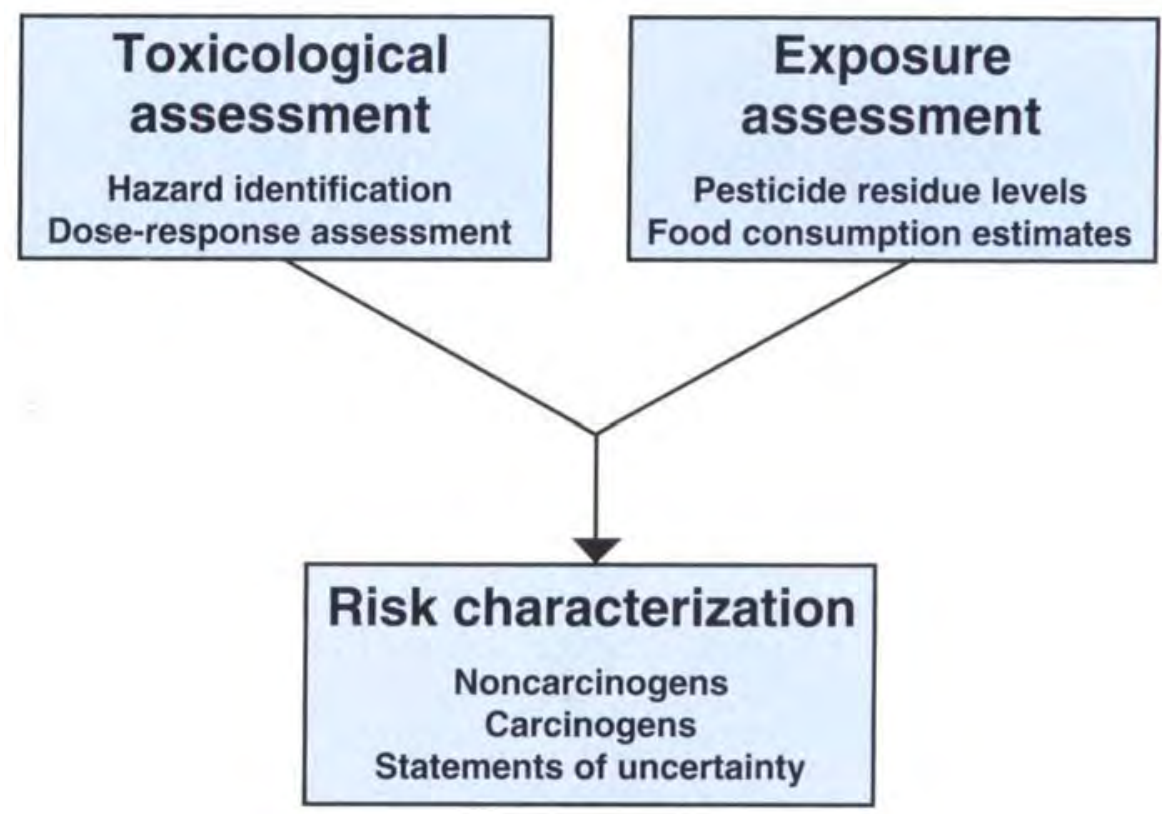

Fig. 1. Basic process of dietary pesticide risk assessment.

timates to be made for pesticide residue levels and food consumption rates. The final step, risk characterization, combines information from the toxicological assessment and exposure assessment phases to yield a risk estimate based on statistical probability. The accuracy of the risk estimate therefore depends upon the accuracy of the toxicological assessment and the exposure assessment, both of which present significant scientific difficulties.

\section{Difficulties in toxicology}

The basic challenge of the toxicological assessment is to identify the most important toxicological effects observed in animal studies and to determine levels of human exposure that would be required to cause the same types of effects. The premise is that the results of animal toxicology studies may be predictive of the potential effects in humans; this premise has been validated in many cases, although there are also several exceptions.

In most cases, few data exist from which to extrapolate the results of animal studies to potential human effects, and several conservative assumptions are made (such assumptions tend to increase the estimate of risk). In the case of noncarcinogenic effects, for example, uncertainty factors (also known as safety factors) are incorporated into the process. The highest dose of a chemical shown not to cause any effect (the no observable effect level, or NOEL) in animal studies is divided by an uncertainty factor to yield an acceptable level of human exposure (often referred to as the acceptable daily intake, ADI, or the reference dose, RfD). An uncertainty factor of 100 is most commonly used. The 100-fold uncertainty factor includes a 10 -fold uncertainty factor to allow for differences in sensitivities between animals and humans multiplied by another 10fold uncertainty factor to allow for susceptibility differences among individual human members of the population. The NAS report recommended that an additional uncertainty factor of up to 10-fold be used in some cases to account for sensitivity differences between infants or children and adults (for a summary of the NAS findings, see p. 15).

Carcinogens (cancer-causing chemicals) present additional challenges in the risk assessment process. In contrast to noncarcinogenic effects, it is often assumed that a threshold level of exposure to carcinogens does not exist. 
This assumption is the subject of intense scientific controversy. It derives in part from radiation biology studies and is based on the observation that many carcinogens are genotoxic that is, they cause direct damage to genes that may lead to cancer. Therefore, any level of exposure to a carcinogen might present some calculable risk of cancer. The risk is still related to the dose, as it is with noncarcinogenic effects.

Since typical human exposure levels to potentially carcinogenic pesticides are often several thousand times lower than the doses that have produced cancer in animal studies, calculation of carcinogenic risks requires extrapolation of the results of highdose animal studies to potential human effects at low levels of exposure. Scientists have adopted mathematical models to predict human effects based on animal effects.

Such models yield a value known as the cancer potency factor. Because a variety of different mathematical models exist, cancer potency factors vary widely depending upon the choice of the model and its assumptions. The most common model used is the linearized multistage model (see p. 26). This model assumes that a cell which may be a target for a carcinogenic chemical goes through a specific number of different stages and that the probability of a "hit" on the cell leading to cancer is stage-specific. At very low levels of exposure, the relationship between exposure level and excess cancers is linear; any finite level of exposure leads to some numerical value of excess cancers (above the background level in the population).
The NAS report recommended use of the two-stage clonal expansion model, itself a recent interpretation of the multistage model. This is a biologically based model of carcinogenesis that takes into account the special physiological characteristics of infants and children, such as the rapid rate of cell division and tissue growth. Biochemical events in infants and children, such as enzymes induction at different stages of development, may affect their response to pesticide residues. In addition, the NAS report recommended development of "pharmacokinetic" models for infants and children - that is, models that describe the child's unique physiological patterns of residue uptake and distribution, allowing for factors such as lactation and development. The report also recommended use of the so-called

\section{Risk assessment defined}

\section{Risk assessment}

Risk assessment is the characterization of the potential adverse health effects of human exposures to substances such as pesticide residues. It also includes characterization of uncertainties inherent in the process.

Risk assessments consist of the following steps:

Hazard identification, which is the determination of whether a particular chemical is or is not causally linked to particular health effects.

Dose-response assessment, which is the determination of the relation between the magnitude of exposure and the probability of occurrence of the health effects in question.

Exposure assessment, which is the determination of the extent of human exposure before or after application of regulatory controls.
Risk characterization, which is the description of the nature and often the magnitude of human risk, including attendant uncertainty.

In each step, a number of decision points occur where risk to human health can only be inferred from the available evidence and judgments must be made regarding the analytic approach to be taken.

\section{Related terms}

Conservative guideline: The term conservative has been used to describe inference options in hazard identification that increase the likelihood that a substance will be judged to be a significant hazard to human health.

Dose-response relationship: This is the relationship between the dose of a chemical substance received and the resulting physiological response. The larger the dose, the greater the effect. (Also, see Threshold.)

Risk management: Risk management is the process of evaluating alternative regulatory options and selecting among them. A risk assessment may be one of the bases of risk management.

Threshold: The minimum dose of a substance that produces a physiological effect. At extremely low doses, most chemical substances are detoxified by the body. Carcinogens are thought to be the exceptions. Because most cancer-causing substances are genotoxic - causing direct damage to genes - any one damaged cell could eventually give rise to a tumor, and any dose is hypothetically capable of causing cancer.

(Some definitions were adapted from Risk Assessment in the Federal Government: Managing the Process [National Academy Press, 1983].) -Editor 


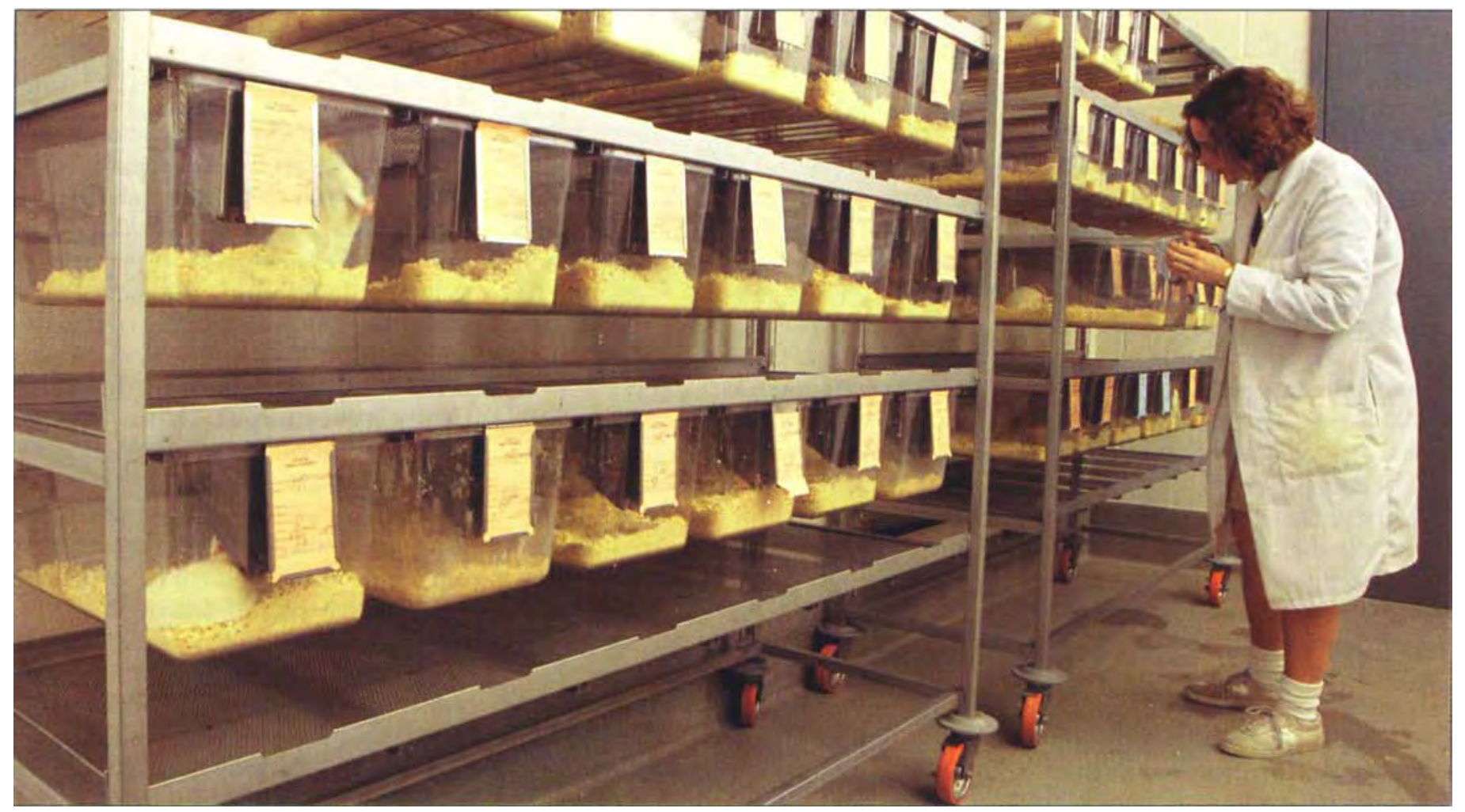

Toxicological effects identified in animals are extrapolated to predict the potential effect of the chemical in humans. When these data are not available, conservative assumptions must be made to estimate risk.

"benchmark dose" in assessing risk to infants and children. The benchmark dose uses all available dose-response data, better reflects the slope of the dose-response curve and provides an explicit indication of risk at doses below the benchmark dose.

\section{Exposure assessment}

To use the information derived from the toxicology assessment phase in risk assessment, it must be combined with an estimate of the likely amount of human exposure, or dose. Two major questions need to be answered during the exposure assessment phase: (1) What are the levels of pesticide residues in various food items? and (2) How much of the various food items are eaten?

Residue estimates. Multiplication of the residue levels by food consumption values for an individual food yields an estimate of the exposure from the particular food; addition of exposures from all possible food items (including water) provides an estimate of total dietary pesticide exposure.
Typical approaches taken in the estimation of residue levels have ranged from those that simply assume that all residues are present at a predetermined level (for example, tolerance levels representing maximum legal residues) to more complex approaches based on actual measurements of residue levels at the time the food is ready to be consumed. A variety of intermediate techniques incorporating data on pesticide use and actual field residue levels may also be used. The choice of method used to estimate residue levels depends upon the availability and reliability of data and the degree of refinement necessary. In many cases, for example, assumptions of residues at the tolerance levels still lead to risks far below those considered to be significant and additional refinement of the residue levels is not warranted.

Some of the methods for assessing pesticide residue levels are shown in figure 2. Approaches requiring the lowest cost often yield the greatest amount of data but provide the greatest overestimation of residue levels. As refinements are made in the process to accommodate additional data, the accuracy of the residue estimates is improved, but the data are less available and the cost to produce additional data rises significantly.
Consumption estimates. The determination of the amount of consumption of particular food items by the population at large or by particular population subgroups is also important in the assessment of dietary pesticide exposure. In the United States, food consumption estimates are most commonly derived from the results of U.S. Department of Agriculture nationwide food consumption surveys. Such surveys involve tens of thousands of individuals in the 48 continental states and Hawaii, Alaska and Puerto Rico. Individuals are asked to describe the types and amounts of foods they consumed, both at home and away from home, for a defined 3-day period.

The adequacy of this approach has been called into question. A major limitation appears to be the sample size for certain subpopulation groups such as nursing infants, which was considered from the latest (1987-88) survey to be too small to confer statistical credibility. Also, the accuracy of surveys of this type is limited by the inability of those surveyed to accurately remember what they consumed during the specified period.

In addition to problems resulting from difficulties in acquiring accurate food consumption estimates from na- 


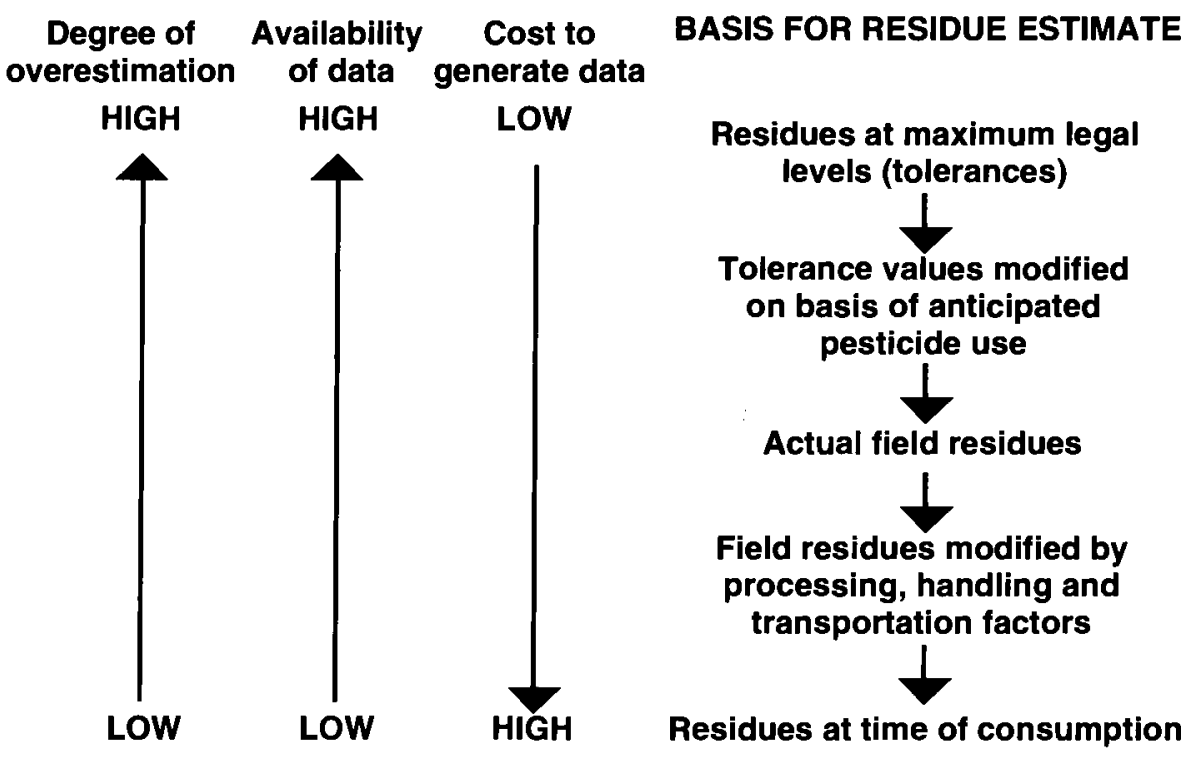

Fig. 2. Various methods of assessing pesticide residue levels.

tional surveys, individual variation in food consumption by members of the same population subgroup is also difficult to account for in the risk assessment process. This is particularly important in determining acute exposure to pesticides in the diet, since shortterm consumption of particular foods may differ dramatically from average, long-term consumption.

To compensate for some of the inaccuracies in food consumption estimation, it is often suggested that average levels of consumption be replaced by consumption estimates of those consuming the greatest amounts of individual food items. This approach may overcome the possibility of underestimating the exposures to some members of the population but may also lead to unrealistic exposure estimates for the general population since it does not allow for compensation in the diet to reduce consumption of other foods.

More accurate estimates could be obtained using "risk distributions," one of the chief recommendations of the NAS report. Risk distributions combine statistical data on the kinds and quantities of foods eaten by children with data on the pesticide residues on these foods. Risk distributions, which show a statistical distribution of risk across a popula- tion, would replace the current method of estimating risk from each individual pesticide using a single number to represent average exposure of the entire population. Risk distributions would help regulators view children in a variety of circumstances, those with above-average and belowaverage exposure.

\section{Interpreting results}

While risk assessment is limited in its ability to accurately calculate human risks from exposure to pesticide residues in the diet, it plays an important and necessary role in the pesticide regulatory process. The use of risk assessment allows regulators to prioritize risks, allocate finite resources, and base regulations upon consistent criteria. It is critical, however, that the limitations and inaccuracies of the risk assessment process be understood if results of risk assessments are to be properly interpreted.

Typical risk assessments rely upon conservative assumptions that increase the risk estimate over one determined using less conservative assumptions. This prudent approach has wide philosophical acceptance as society tends to agree that if errors are to be made, it is best to err on the side of safety. Thus, the risk estimates them- selves may overstate actual human risks, as a deliberate public policy.

While the findings of the NAS committee may indicate that increased regulatory controls or morerefined risk assessment techniques are necessary, they should not be taken as indicators of immediate health risks. Conservative assumptions and uncertainty factors built into the process afford a margin of safety.

In the case of carcinogen risk assessment, care should be taken to avoid misinterpreting results through "body count" analyses in which risk estimates are multiplied by population numbers to suggest "actual" cases of human cancer. Significant uncertainty is factored into the process through the use of conservative mathematical models; the actual cancer risks may be significantly lower or even zero.

\section{Scientific challenges}

There is no doubt that significant scientific advances are needed to improve the accuracy and utility of risk assessments. It should be emphasized that toxicology itself is a young and evolving science and that risk assessment is only in its formative stage of development.

The recent NAS report made several suggestions for improving the accuracy of dietary pesticide risk assessment (see p. 15). It is likely that adoption of many of the NAS recommendations could significantly improve the accuracy of the risk assessment process.

Over the past several years, much of the scientific community has maintained that improvements are particularly needed in the assessment of the risks from potentially carcinogenic pesticides. Carcinogen risk assessment receives a disproportionate level of attention and detracts from the assessment of other types of risks such as nervous system, developmental, immune system and reproductive effects (see p. 17). This overemphasis stems primarily from the unique treatment that potential carcinogens receive concerning the lack of a toxicity threshold level and the use of particucontinued on p. 27 


\section{How does cancer begin?}

\section{A brief description of the multistage model of cancer}

\author{
Andy Salmon
}

$\mathrm{W}$ Then an organism is exposed to a toxic chemical, the nature and extent of the damage depends on the nature of the chemical and extent of the exposure (at what concentration, for how long). Usually, the effect of a chemical on an individual is progressive: increased exposures cause increased damage. In the case of cancer however, either the subject has a tumor or does not; there are no half measures. What changes with the dose of a carcinogenic chemical is not the severity of the response, but the chance that it will occur. Thus, for carcinogens the dose-response relationship is decribed in terms of risk associated with exposure to a certain level of a chemical for a certain amount of time.

The most widely accepted mathematical model for cancer is the "multistage model of carcinogenesis." Its basic hypothesis is that cancer results from alterations in growth-regulatory genes, so that affected cells are no longer subject to the strict control of growth and differentiation of normal tissue cells. The observed process has three phases:

- Initiation, in which some genetic alteration occurs in a target cell,

- Promotion, in which the altered cell grows and develops a clone group of altered cells appearing as a tumor,

- Progression, in which the tumor cells grow, become more aberrant and invade surrounding tissues; this requires a series of secondary genetic changes.

The multistage model of human cancer is based on the idea that several successive mutational events are necessary to result in the disease. Current research on human tumor development suggests that five or more different mutations may be required to generate malignant tumor cells. This helps explain why cancer is associated with age.
Genetic changes, which can be produced by reactive chemicals damaging the DNA, are the essential feature of the initiation phase, and are also thought to be involved in the later stages. To model what is known about the carcinogenic process, researchers assume there is no threshold dose. Any exposure to a toxicant that can damage DNA involves some risk of contributing to the carcinogenic process. Because cancer develops over time, and through multiple stages, the risk posed by single carcinogenic exposures is likely to be very small. However, continued exposures to carcinogens over time is statistically associated with disease. More recent research suggests that the multi-stage progression is actually a series of cycles of genetic damage followed by cell growth. In this case, certain exposures that interfere with this cycle at later stages could be just as damaging as those causing initiation.

Based on what is known about the relationship between DNA damage and cancer induction, researchers also assume that the relationship between dose and carcinogenic response is linear at low doses. At higher doses (such as those in animal cancer bioassays) the response curve may become steeper. Theory suggests that added exposures over time actually may have a multiplicative, rather than a simply additive, effect on probability of disease. Repeated low-dose exposures over a lifetime can, therefore, significantly increase probability of disease. This is in contrast to threshold models commonly used to assess noncarcinogenic risks in which the damage process only begins when some protective factor or process has been exceeded.

Most of the documented examples of human cancer induced by chemical exposures involved repeated exposures over time and across disease pro- gression stages. Similarly, most animal studies involve prolonged exposures, usually for most of the expected lifetime of the test animals. One important point of the multistage model, which is supported by experimental findings in human and animal studies, is that while high-dose, short-term exposures to carcinogens are an appropriate public health concern, so too are lower-dose exposures that can contribute to cumulative genetic damage over long periods of time. Particularly given a consistent background of carcinogenic exposure, the issues of multistage development and the multiplicative effect of repeated exposures make examination of and prevention of exposure to any carcinogens a public health concern.

Extended mathematical models have been developed to help analyze these situations. The "two-stage clonal expansion model" proposed by Moolgavkar and co-workers of the Division of Public Health Sciences, Fred Hutchinson Cancer Research Center in Seattle, may do a better job of predicting promoter effects by taking into account the processes of cell death and cell division. The long-term effects of these processes may be particularly important in infants and children, whose tissues are undergoing high rates of cell division as a natural part of maturation. Also, the long period of expected lifespan following an exposure during infancy may increase the chances of seeing adverse effects with a very long latency (the time between the initial exposure and the observation of the final result, such as a tumor).

A. Salmon is Staff Toxicologist for the Reproductive and Cancer Hazard Assessment Section of the California Environmental Protection Agency. 
Assessment (continued from p. 25)

lar mathematical models that incorporate nonthreshold behavior. While it is extremely difficult to determine experimentally whether carcinogens do or do not act in a threshold dependent manner, many view the assumption that carcinogens may not be subject to threshold doses to be overly conservative.

Another point of controversy surrounding carcinogen risk assessment involves toxicology testing protocols. Typically, massive levels of chemicals (see p. 28) are given to animals throughout their lifetimes at the maximum tolerated dose (MTD), which is typically several orders of magnitude higher than levels humans would expect to receive. It has been argued that cancers produced at the MTD may result from cell death and errors in subsequent cell replication caused by the high doses and would not be expected at lower, more realistic doses. In addition, results of cancer studies are often equivocal, and many chemicals may be considered as carcinogens even though a majority of studies have been negative for carcinogenicity.

The conservative "nonthreshold" approach for carcinogens also ignores significant mechanistic developments in toxicology that have shown that chemicals may produce cancer by a variety of means. In several cases, chemicals classified as potential carcinogens have not been identified as genotoxic (mutagenic) agents; mounting evidence for many of these chemicals indicates that their carcinogenic effects may require exposure in excess of a toxicity threshold. Thus, it is likely that the use of a single mathematical model may not be applicable to all chemicals.

\section{Regulatory, policy implications}

Dietary pesticide risk assessments are commonly used to determine if pesticides may be registered for use on particular commodities. The Environmental Protection Agency (EPA), under provisions of the Federal Insecticide, Fungicide, and Rodenticide Act, has the authority to approve pesticides for use if the benefits of the intended uses are determined to outweigh the risks. The determination of dietary

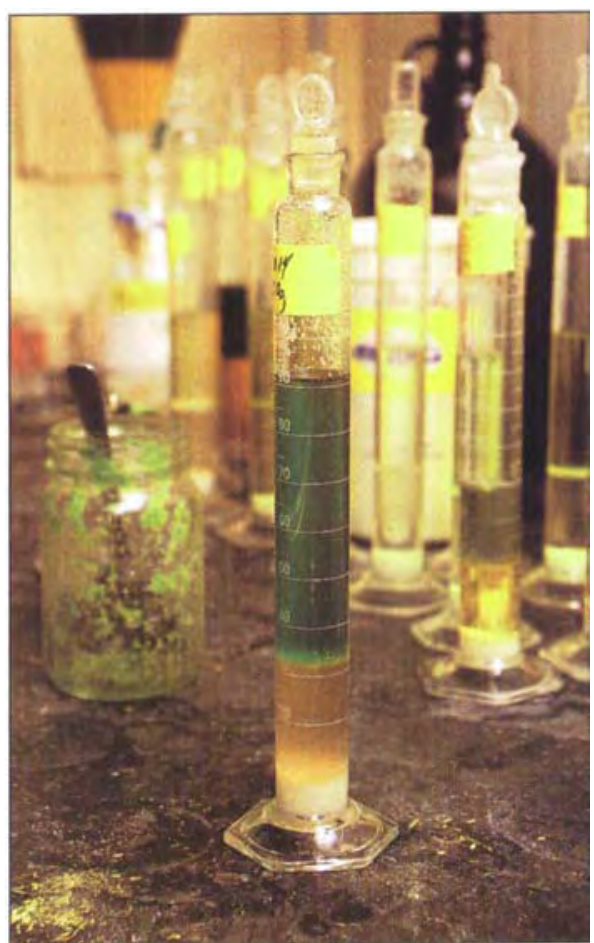

risks from pesticides is a critical component of EPA's overall risk assessment process, and may have significant effects upon pesticide use practices in the United States. It is important that the risk assessment decisions made by EPA be scientifically sound, because mandated changes in pesticide use have wide effects on the nation's agricultural economy, including food availability and cost. While public health should be government's first priority, policy makers also need to be cognizant of economic impacts on the nation's significant industries.

When introduced into the regulatory and policy arenas, the ultimate "number" generated from the risk assessment may take on exaggerated importance while appreciation of the imprecision and complexities of the risk assessment process tends to fade. The uncertainties of risk assessment do not lend themselves well to politics, and policies are often developed through use of a "bright-line" approach to determine, in absolute terms, the acceptability of specific risks. Such an inflexible approach may neglect the benefits of specific chemicals or the alternative risks from not using the chemicals. As an example, risk assessments have demonstrated that specific fungicides may present potential carcinogenic risks. Restrictions on fungicide uses would minimize these risks, but may lead to other risks, since fun- gicides control the production of naturally-occurring carcinogenic mycotoxins. More importantly, the inflexibility may not allow future scientific advances to be incorporated into risk assessment practices. As the scientific understanding of the mechanisms for chemical carcinogenesis increases, new mathematical models may allow the risks to be calculated more accurately.

Delaney Clause. As an example, a recent court decision upheld a strict interpretation of the Delaney Clause of the Federal Food, Drug, and Cosmetic Act; this action has forced EPA to begin cancellation procedures for several food-use pesticides (see p. 30). While many uses of pesticides are not subject to regulation as food additives, several others are affected by the "zero-risk" standard of the Delaney Clause. Removal of these pesticide uses based upon statutory grounds will provide trivial health benefit to consumers but could cause significant effects upon domestic and international agriculture.

Significant effort is being made in Congress to replace the Delaney Clause with new legislation. In place of the "zero-risk" provision of Delaney, the concept of "negligible risk" is being considered. Different bills prescribe different methods for the determination of what constitutes a negligible risk and how risks should be calculated. Ironically, these risk assessment prescriptions may also be considered to be inflexible and may not allow future scientific improvements in risk assessment to be used. The legislation, if adopted, may appear to be as scientifically obsolete in 35 years as the Delaney Clause appears to be today. It is critical that legislation allow pesticide regulators to make use of the best available scientific practices rather than prescribed, outdated methods.

NAS recommendations. There is no doubt that incorporation of many of the NAS recommendations will improve the scientific basis for assessing human risks from pesticide residues in the diet. At the same time, however, major assumptions are still required and future risk assessments will still be limited in their accuracy. The costs continued on $p .29$ 


\section{Debate simmers ...}

\section{Should carcinogens be tested at maximum tolerated dose?}

\section{Gail Charnley}

$\mathrm{W}^{-1}$ hen chemicals are tested in laboratory animals to determine if they can cause cancer, they are tested at the maximum tolerated dose (MTD). The MTD is defined as the highest dose that can be administered to animals on a long-term basis that does not reduce their lifespans from causes other than cancer. MTDs are frequently much higher than the doses of chemicals that humans receive from environmental exposure. When chemicals are tested at such high doses, they can elicit physiologic alterations, including increased rates of cell proliferation, that would not be expected to occur at the lower doses to which humans are generally exposed. For example, a chemical that causes DNA damage will magnify the effects of that damage if its toxicity at high doses leads to increased cell proliferation. In this case, the chemical will appear to be a more potent carcinogen at high doses than it would be if it were tested at low doses.

The potential discrepancy between mechanisms of carcinogenesis operative at high doses and those operative at lower doses raises questions regarding the relevance of carcinogenicity testing at the MTD. Are chemicals identified as carcinogens only at high doses relevant to human exposure? Without some knowledge of the biologic mechanisms by which a chemical induces cancer at high doses, this is a difficult question to answer.

Cell proliferation plays a critical role in the development of cancer. When a cancer-causing agent reacts with a site on a cell's DNA (or genetic material), the cell may or may not be able to repair itself. If not, the DNA may be altered such that when it is copied prior to cell division, its sequence is changed permanently in the new cell. This change is called a mutation. If the mutation occurs at a site that is critical for controlling the cell's rate of proliferation or differentiation, the cell may be transformed into a cancer cell, which in turn can proliferate further and appear as a tumor. Increasing the rate of cell proliferation can reduce the time that a cell has to repair damage to DNA and thereby increase the likelihood that mutations will occur.

In general, the rate at which a cell proliferates can be increased in one of two ways. Some chemicals, called mitogens, can increase cell division by interacting with special receptors on a cell's surface that send signals to the sites on DNA that control proliferation. Other chemicals can be toxic to a group of cells, and increased cell division occurs as a consequence of toxicity as the remaining cells attempt to replace those that were killed.

The specificity and sensitivity of carcinogenicity tests in animals are important considerations for evaluating whether the results of animal tests can be used to predict a chemical's behavior in humans. "Sensitivity" refers to the ability of animal tests to detect true human carcinogens, and "specificity" refers to their ability to avoid identifying chemicals as human carcinogens when they are not. The sensitivity of animal tests appears to be high; all of the known human carcinogens tested have been positive in at least one animal species, although the data for target organs are often inconsistent. The specificity of animal tests cannot be evaluated at present, however, because available data on human noncarcinogens are insufficient to make comparisons. In the absence of information to the contrary, the assumption is made by regulatory agencies that evidence supporting or refuting carcinogenic activity in animal studies should be considered applicable to humans until better information is obtained.

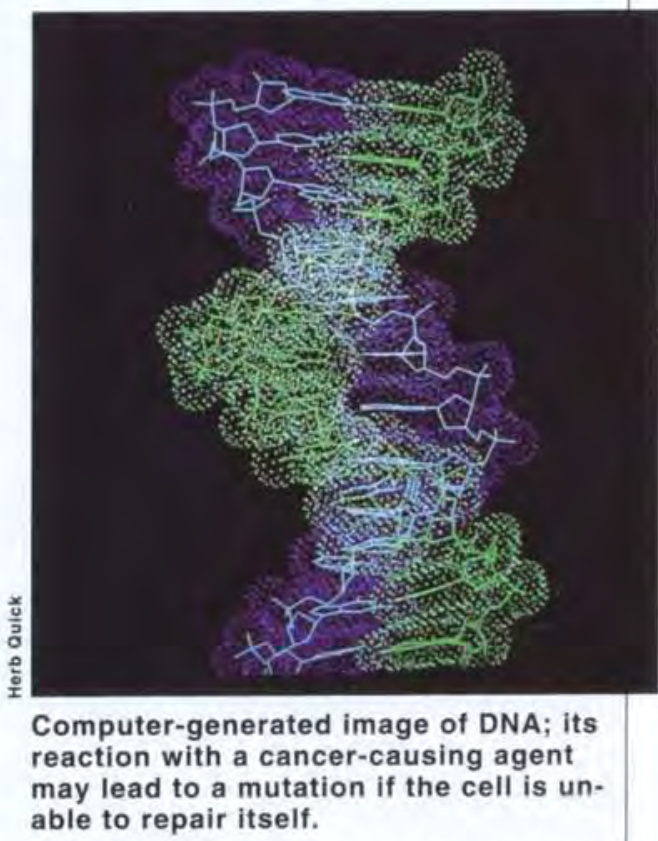

There is controversy in the scientific community regarding the use of the MTD. One view is that the MTD should continue to be used to screen chemicals for potential carcinogenicity because using lower doses could sacrifice the sensitivity of animal tests; that is, fewer chemicals would be identified as carcinogens at lower doses, and a potential human carcinogen could be missed. The other view is that instead of using the MTD, the highest dose tested should be that which elicits effects comparable to those expected if actual human exposure is taken into account. This would increase the specificity of animal tests. Choosing the dose that is most relevant to humans is likely to be difficult without knowledge of each chemical's biologic mechanisms of action, however. Understanding a chemical's biologic mechanisms of action requires more extensive testing than is generally performed at present.

G. Charnley is Toxicologist and Senior Staff Scientist for the Board on Environmental Studies and Toxicology of the $\mathrm{Na}$ tional Research Council, the research arm of the National Academy of Sciences. 
Assessment (continued from p. 27)

associated with implementing many of the NAS recommendations need to be considered as well, since processes such as standardizing residue determination practices, improving food consumption estimates and incorporating earlier stages of animal development into toxicological testing protocols may involve significant expenditures. It is critical that such expenditures be balanced against possible health benefits that could be derived from implementation of such practices. At the present time, data indicate that for most pesticides, even under the exaggerated assumptions that they are always present at the maximum allowable levels, typical human exposure is well below established health criteria such as the ADI or RfD. Rather than employ state-of-the-art risk assessment techniques to all pesticides across-the-board, it seems prudent to initially use less sophisticated analyses to prioritize pesticide uses of greatest concern and reserve the more sophisticated models for the most important pesticides.

Priorities in perspective. While we need to improve the risk assessment process, and protect all subgroups of the population, we must keep pesticide residue concerns in proper perspective. The driving force behind pesticide legislation and regulation today seems to be food safety, although most would contend that environmental effects from pesticides and worker safety issues are much more important. In terms of overall food safety, pesticides are considered by the U.S. Food and Drug Administration as a fifth priority, of less concern than (1) microbiological contamination of foods, (2) nutritional imbalance, (3) environmental contaminants and (4) naturally-occurring toxins. Pesticide food safety issues should not be ignored, but should be considered at an appropriate level when precious resources are allocated and health policies are formulated.

C. K. Winter is Director, FoodSafe Program, and Extension Food Toxicologist, Department of Food Science and Technology, UC Davis.

\section{Congress considers pesticide reform proposals}

工 egislation now in Congress attempts to address issues raised by the National Academy of Sciences report Pesticides in the Diets of Infants and Children (see p. 15) and the recent federal court ruling strictly enforcing the Delaney Clause (see p. 30). If passed, such legislation would affect provisions of the Federal Food, Drug and Cosmetic Act (FFDCA) and Federal Insecticide, Fungicide and Rodenticide Act (FIFRA).

Currently Congress is considering two pesticide reform bills:

- HR 1627 sponsored by Richard Lehman(D-CA), Thomas Bliley (D-GA) and Roy Rowland (R-VA) and

- S 331/HR 872 introduced by Edward Kennedy (D-MA) and Henry Waxman (D-CA).

HR 1627 allows for a flexible, negligible risk standard based on science and consideration of pesticide benefits in making regulatory decisions. It also expedites the removal of harmful pesticides from the market.

The Kennedy/Waxman bills seek to strengthen the EPA's authority to set tolerance levels of negligible risk, addressing the unique susceptibility of children to pesticides, and force raw foods to meet the same safety standards as processed food. They would also simplify the procedures for removing unsafe pesticides from the market, eliminate consideration of economic benefits and establish a mandatory timetable for determining whether a pesticide is safe.

Compelled by charges that the government needs to do more to protect the health of its citizens, the Clinton Administration has proposed its own pesticide safety reform package.

The Administration's reform plan recommends amending the law to set tolerances "based on a strong health-

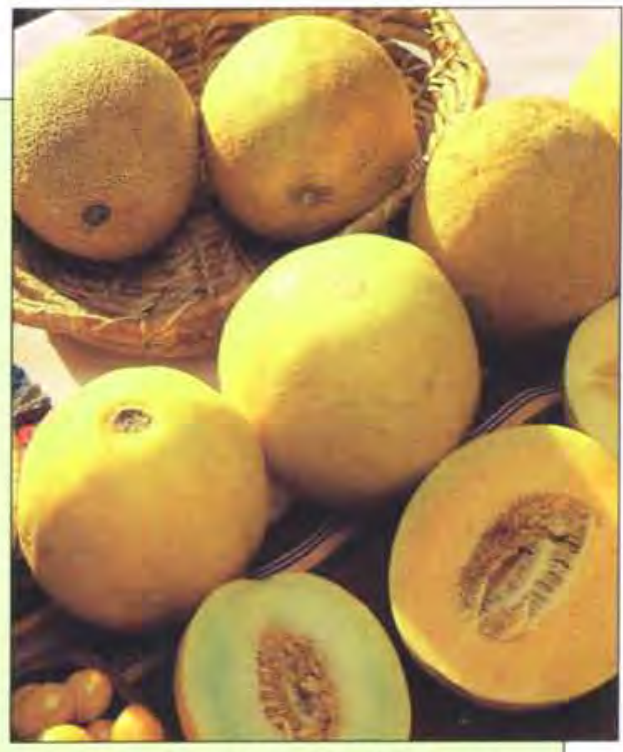

One pesticide reform bill in Congress would force raw foods to meet the same safety standards as processed foods.

based safety standard of reasonable certainty of no harm to consumers of food." For carcinogens, it would require negligible risk. Addressing recommendations in the NAS report Pesticides in the Diets of Infants and Children, the plan states tolerances would also have to be protective for children and EPA would consider multiple exposures and children's food preferences when establishing tolerances.

Among enforcement provisions of the plan, EPA would be required to complete review of all existing tolerances to ensure that they meet the safety standard within 7 years of enactment. A special "fast track" review process would be started for pesticides that appear not to satisfy the safety standard.

The law requiring safe pesticide residue tolerances be set for processed foods was written 36 years ago. In this law, the Delaney Clause prohibits food additives shown to cause cancer. Because technological advances over the years have enabled scientists to detect the presence of pesticides at lower and lower levels, the law has been enforced on the basis of negligible risk, rather than zero risk. (Negligible risk is usually defined as the potential to cause one additional cancer in a million people.) However, in 1992, a federal court ruled that the EPA should strictly enforce the Delaney Clause, $-E d$. 